\title{
Comparación del abordaje sublabial transesfenoidal microquirúrgico frente al abordaje endonasal transesfenoidal endoscópico. Estudio prospectivo de 50 pacientes
}

\author{
J. Enseñat; J.L. Quesada*; J. Aparicio**; C. Pàmies***; X. Barber**; Th. Topczewski y E. Ferrer
}

Servicio de Neurocirugía, Hospital Clínic i Provincial. Barcelona. Servicio de Otorrinolaringología, Hospital Valle de Hebrón. Barcelona*. Departamento de Estadística, matemáticas e informática, Universidad Miguel Hernández. Elche**. Servicio de Anestesiología y Reanimación, Hospital Sant Joan de Déu. Esplugues de Llobregat***.

Resumen

Objetivos. Comparar el abordaje transesfenoidal endonasal endoscópico con el abordaje transesfenoidal sublabial microquirúrgico. Valoración del grado de resección tumoral, grado de invasividad tumoral, complicaciones y estancia postoperatoria de ambos grupos.

Material y métodos. Estudio prospectivo no randomizado de 50 pacientes intervenidos en nuestro centro entre 2002 y 2006 de lesiones en la región selar con diferentes grados de invasión del seno cavernoso según la clasificación de Knosp. Entre las variables a estudio se incluyeron los grados de invasión, los grados de resección postoperatoria (total, subtotal y parcial), lesión del nervio óptico, panhipopituitarismo postoperatorio, fístula de LCR, déficit de pares craneales, epistaxis, meningitis, diabetes insípida y lesión de arteria carótida. Nuestra serie consta de 27 hombres y 23 mujeres, con una edad media de 48 años (19-80 años). En 23 casos se utilizó una vía transesfenoidal sublabial microquirúrgica ( 2 pacientes fueron excluidos) $y$ en 25 casos se realizó un abordaje transesfenoidal endoscópico. El seguimiento medio fue de 12 meses.

Resultados. En nuestra experiencia la técnica endoscópica presenta un porcentaje de exéresis completa superior al de la técnica clásica $(60 \%$ frente a $34,8 \%$ ) que también se hace evidente en el caso de la resección subtotal $(32 \%$ frente $26 \%)$ existiendo una diferencia estadísticamente significativa $(p=0,033)$. En contraposición no encontramos diferencias en cuanto a complicaciones. También hemos evidenciado que existe una diferencia estadísticamente significativa en cuanto a la estancia postoperatoria $(p=0,001)$, reduciéndose ésta a la mitad (3 días) con la técnica endoscópica. Si bien no hemos encontrado diferencias significativas en cuanto al grado de invasividad tumoral y al grado de resección, un mayor grado de invasividad aumenta en 3,59 veces el riesgo de poco éxito de la cirugía.

Discusión y conclusiones. En nuestra experiencia

Recibido: 3-10-08. Aceptado: 2-12-08 con la técnica endoscópica obtenemos un mayor grado de resección quirúrgica y una estancia postoperatoria menor. No hemos observado diferencias en cuanto a complicaciones.

PALABRAS CLAVE: Tumores hipofisarios. Abordaje transesfenoidal sublabial. Abordaje transesfenoidal endoscópico. Complicaciones en cirugía hipofisaria. Grados de Knosp.

Prospective comparative study on 50 patients between microsurgical sublabial transsphenoidal approach and endoscopic endonasal transsphenoidal approach

\section{Summary}

Objective. Compare the standard transsphenoidal sublabial microscopic approach with the endoscopic transsphenoidal approach concerning the tumoral invasiveness and resection, complications of the approaches and time of post operative hospitalisation.

Material and methods. We realized a prospective, non randomised study with 50 patients. They were operated between 2002 and 2006. All the patients had sellar lesions with different grades of invasiveness of the cavernous sinus as classified by Knosp. The variables included in our study were tumoral invasiveness and operative resection (total, subtotal and partial), optic nerve lesion, postoperative panhypopituitarism, CSF fistula, cranial nerves deficits, epistaxis, meningitis, diabetes insipidus and carotid artery lesion. Our series included 27 males and 23 females ranging from 19 to 80 years old (48 mean). In 23 patients we used the standard sublabial microscopic approach (two patients were excluded) and for 25 patients we used the endoscopic approach. The mean follow up was of 12 months.

Results. In our experience the endoscopic technique presents a higher percentage of total resection comparing to the sublabial microscopic approach $(60 \%$ versus $34,8 \%$ ) and higher percentage of subtotal resections $(32 \%$ versus $26 \%)$ with a statistical significant 
difference $(p=0,033)$. The time of hospitalisation was significant shorter for the endoscopic approach group $(p=0,001)$, diminishing by half of the time ( 3 days) of the microscopic approach group. Concerning the tumoral invasiveness and complications we did not appreciate any significant dissimilarity. We appreciated that a higher grade of invasiveness augments by 3,59 the risk of an unsuccessful surgery.

Discussion and conclusion. In our experience the endoscopic technique may favour a better tumoral resection and shorter time of hospitalisation. We did not appreciate differences concerning the complications.

KEY WORDS: Pituitary tumors. Transsphenoidal sublabial approach. Transsphenoidal endoscopic approach. Complications in pituitary surgery. Knosp grades.

\section{Introducción}

El abordaje transesfenoidal es y ha sido una de las piedras angulares de la neurocirugía para el tratamiento de los tumores hipofisarios y de su región adyacente. El auge de los abordajes endoscópicos a la región selar ha ido substituyendo, no sin polémica, al clásico abordaje transesfenoidal. Desde que $\mathrm{Jho}^{20}$ estandarizó la técnica, ésta ha ido extendiéndose y perfeccionándose en estos últimos años ${ }^{5}$ por autores como Cappabianca, Kassam y Frank. El auge de la endoscopia en la cirugía transesfenoidal viene dado por una serie de condicionantes previos favorables. Uno de ellos es el hecho de que las fosas nasales son una cavidad real que no requiere dilatación para entrar y con acceso directo a la silla turca. La otra gran ventaja de la endoscopia transesfenoidal es que ofrece una gran capacidad y variabilidad de posibilidades tanto en los sistemas de visualización como en la utilización de instrumental quirúrgico. Como ejemplos destacar que el abordaje endoscópico puro se puede realizar a través de un agujero nasal o de los dos; y que podemos utilizar o no un brazo que sujete el endoscopio o bien trabajar con el espéculum nasal ${ }^{9,10,18,19,30}$. Los endoscopios utilizados son rígidos con angulaciones de 0,30 y 45 grados permitiendo la visualización de regiones que anteriormente con el microscopio eran de difícil acceso. Algunos autores también utilizan los endoscopios flexibles ${ }^{22}$ como complemento al microscopio, o endoscopios rígidos para completar la cirugía principalmente en tumores supraselares.

Nuestro objetivo es describir la experiencia de un grupo joven, no experto en cirugía hipofisaria y que optó por iniciarse en la técnica endoscópica. Creemos firmemente que la adopción de nuevas técnicas no tendría que comportar un alto precio para nuestros pacientes. Este estudio nació y se realizó para evaluar la eficacia y seguridad que tenía una nueva técnica en nuestras manos.

\section{Material y métodos}

Estudio prospectivo no randomizado. Analizamos 50 pacientes consecutivos, hombres (27) y mujeres (23) entre 19 y 80 años de edad (media 48.02), que fueron hospitalizados entre 2002 y 2006 en el hospital del Valle de Hebrón de Barcelona. Todos los pacientes fueron intervenidos por un mismo neurocirujano (JE). Todos los pacientes a los que se les realizó un abordaje endoscópico fueron intervenidos conjuntamente por un neurocirujano y un otorrinolaringólogo (JE, JLQ). La mayoría de los pacientes (39) presentaban un macroadenoma, 3 pacientes presentaban un quiste de Bolsa de Rathke y 8 fueron intervenidos quirúrgicamente de un microadenoma hipofisario. El seguimiento fue de 1 año.

\section{Estudio preoperatorio}

Todos los pacientes fueron evaluados según criterios clínicos, radiológicos, y endocrinológicos. Clínicamente se diferenciaron los pacientes con debut clínico agudo o subagudo (apoplejía hipofisaria) de los pacientes con sintomatología neurológica y/o endocrina más insidiosa. En todos los casos se realizaron campimetrías computarizadas SITA 24-2 para definir el grado de afectación del campo visual. Endocrinológicamente se evaluó la integridad del eje hipotálamo-hipofisario mediante la determinación de las concentraciones plasmáticas de FSH, LH, ACTH, cortisol plasmático, GH, Prolactina, TSH, T4 libre, estradiol (mujeres) y testosterona (hombres).

El estudio neurorradiológico incluyó en todos los casos un TC helicoidal de las fosas nasales y una RM cerebral de 1.5 T. En la RM se evaluó la invasión del seno cavernoso de acuerdo con la clasificación de Knosp et al. ${ }^{23}$. Estos autores proponen la clasificación de los tumores que invaden el seno cavernoso en cinco grados $(0,1,2,3$ y 4$)$ de acuerdo con los siguientes criterios:

Grado 0: el tumor no invade el seno cavernoso. Todas las estructuras anatómicas intracavernosas están preservadas. El tumor no sobrepasa la línea tangencial que une la pared medial de la arteria carótida interna supracavernosa con la arteria carótida interna intracavernosa.

Grado 1: el tumor sobrepasa la tangente medial (definida como la tangente que une los dos bordes mediales de la carótida supra e intracavernosa) pero no sobrepasa la línea tangencial que une los dos centros de la carótida supra e intracavernosa. Los compartimentos venosos superior e inferior pueden estar obstruidos según el crecimiento de la lesión sea en sentido superior o inferior respectivamente.

Grado 2: Se caracteriza porque el tumor se extiende por detrás de la línea intercarotídea, sin sobrepasar la tangente lateral de la carótida intra-supracavernosa.

Grado 3: el tumor se extiende lateralmente a la línea tangencial lateral que une la porción carotídea supracaver- 
nosa con la intracavernosa. Los compartimientos venosos medial, superior e inferior suelen estar parcial o completamente obstruidos.

Grado 4: se caracteriza porque la carótida está totalmente englobada por el tumor, estando todos los compartimientos venosos obliterados.

\section{Técnica quirúrgica}

Se realizó un abordaje sublabial transesfenoidal clásico en los primeros 23 pacientes. En los siguientes 25 pacientes se realizó un abordaje transesfenoidal endonasal endoscópico descrito por $\mathrm{Jho}^{20}$.

\section{Estudio postoperatorio}

Todos los pacientes fueron estudiados desde el punto de vista clínico, radiológico y endocrinológico (siguiendo los mismos parámetros evaluados preoperatoriamente). La primera valoración se efectuó a los tres meses del alta hospitalaria, y después al año. Nosotros hemos valorado el estado clínico y radiológico al año de la intervención quirúrgica, que es cuando hay una mayor estabilidad en el eje hipotálamo-hipofisario. De acuerdo con los hallazgos de la RM postoperatoria se clasificaron los pacientes en las siguientes categorías: 1) resección completa, cuando no había evidencia de restos tumorales visibles en la RM de control; 2) resección subtotal cuando se consiguió una resección de más del $80 \%$ del tumor inicial y 3) resección parcial en el caso de una resección inferior al $80 \%$ del volumen tumoral. Clínicamente se evaluaron los pacientes en tres categorías: 1) el paciente empeoró su clínica después de la cirugía, 2) no hubo cambios, 3) mejoró su clínica preoperatoria.

Los criterios de curación endocrinológicos fueron:

- acromegalia: una GH inferior a $1 \mathrm{ng} / \mathrm{ml}$ después de la sobrecarga oral de glucosa con IGF-1 normales según la edad.

- prolactinomas: PRL $<30 \mathrm{mg} / \mathrm{dl}$ en mujeres y $<15 \mathrm{mg} / \mathrm{dl}$ en hombres.

- enfermedad de Cushing: cortisol en sangre (matinal) $<50 \mathrm{nmol} / 1$ y nivel de cortisol libre urinario normal.

\section{Variables}

Hemos estudiado las variables edad, grados de Knosp, control por RM de la resección y control clínico. También se han valorado las complicaciones lesión de los nervios ópticos, déficit de pares craneales, panhipopituitarismo, fístula de LCR, epistaxis, meningitis, diabetes insípida y lesión de arteria carótida. Para cada complicación: 0) ausencia y 1) presencia.

Estas variables constituyen el núcleo central del estudio y se ha trabajado sobre éstas para la determinación de las pertinentes relaciones con el método quirúrgico. No obstante, ha sido necesaria tanto la agrupación de algunas de estas variables, como la recodificación de la variable grados Knosp y grado de resección, con el propósito doble de, por un lado, mejorar la valía de los modelos estadísticos ajustados en la investigación y, por otro lado, hacer frente al problema de tamaños de muestra cero para algunas de las categorías de las variables no cuantitativas. A este respecto, hemos agrupado las ocho variables relativas a complicaciones (lesión de los nervios ópticos, panhipopituitarismo, fístula de LCR, déficit de pares craneales, epistaxis, meningitis, diabetes insípida y lesión de arteria carótida) en una única variable que representa la suma de todas éstas. A esta variable la hemos denominado "Complicaciones". Por otra parte, hemos recodificado grados de Knosp en dos únicas categorías: "Leve/Moderada", asociadas a los niveles originales de 0 a 3, y "Grave", asociada al nivel más alto (grado 4) de la variable grados de Knosp. A esta variable la hemos denominado Grados Knosp 2. En cuanto a la variable original grados de resección, hemos tenido que recodificarla en dos categorías: "Total/Subtotal" frente a "Parcial". A esta última variable la hemos denominado en la investigación grado de resección 2 .

\section{Análisis descriptivo}

Se realizó un estudio descriptivo (gráfico y analítico) para la detección de errores de introducción de datos, así como para conocer la caracterización de la población a estudio. Destacar la detección de un outlier dentro del grupo de pacientes intervenidos mediante la técnica clásica. Este paciente presentó un comportamiento en el número de días de postoperatorio (35 días en concreto) que no se corresponde con el patrón promedio observado en el resto de la muestra de datos (la media del número de días de postoperatorio para los pacientes del grupo clásico sin el outlier es de 6.61, y el máximo observado para este mismo grupo con el outlier es de 12 días). En el grupo clásico un paciente fue éxitus con lo que se perdió el seguimiento. En consecuencia, el tamaño final de la muestra para el grupo intervenido mediante la técnica clásica fue de 23 pacientes, frente a un tamaño final de la muestra para el grupo operado mediante la técnica endoscópica de 25 pacientes.

\section{Análisis univariante y multivariante}

Las relaciones entre el método quirúrgico aplicado y las variables de naturaleza categórica del estudio (grados de Knosp, lesión de los nervios ópticos, panhipopituitarismo, fístula de LCR, déficit de pares craneales, epistaxis, meningitis, diabetes insípida, lesión arteria carótida, grado de resección y control clínico) se realizaron mediante tablas de contingencia y el cálculo del test Chi-cuadrado para contrastar la hipótesis de independencia de las variables en unos casos, u homogeneidad, en otros. Para el caso de tablas de contingencia $2 \mathrm{X} 2$ con valores esperados inferiores a 5 en alguna celda, se realizó el cálculo del test exacto de Fisher 
Tabla 1

\begin{tabular}{|l|c|c|}
\hline Manifestaciones clínicas & Microquirúrgico & Endoscópico \\
\hline Déficit visual & 15 & 13 \\
\hline Clínica de acromegalia & 4 & 2 \\
\hline Impotencia & 2 & 3 \\
\hline Clínica de enf. Cushing & 1 & 1 \\
\hline Incidental & 1 & 1 \\
\hline Déficit pares craneales & 0 & 2 \\
\hline Clínica hiperprolactinemia & 0 & 1 \\
\hline Diabetes insípida & 0 & 2 \\
\hline
\end{tabular}

para contrastar igualmente la hipótesis de independencia de las variables. En cuanto a la medición de la relación entre el método quirúrgico y las variables de naturaleza cuantitativa (edad y número de días de postoperatorio), ésta se realizó mediante un test $\mathrm{T}$ para dos muestras independientes, previamente chequeando la hipótesis de igualdad de varianzas entre grupos (clásico/endoscópico) a través de la prueba de Levene. Asimismo, las pruebas de normalidad de las variables de naturaleza cuantitativa se realizaron mediante la prueba no paramétrica de Kolmogorov-Smirnov.

La estimación de las Odds Ratio y sus intervalos de confianza del $95 \%$ de la variable de interés grado de resección 2 frente al método quirúrgico y a la invasión inicial del tumor (variable grados Knosp 2) se realizó mediante una Regresión Logística No-Condicionada, siendo la variable dependiente: eliminación del tumor total o subtotal $(>80 \%)$, frente a eliminación parcial del tumor.

El nivel de significación utilizado a lo largo del estudio es de un $5 \%(\alpha=0.05)$.

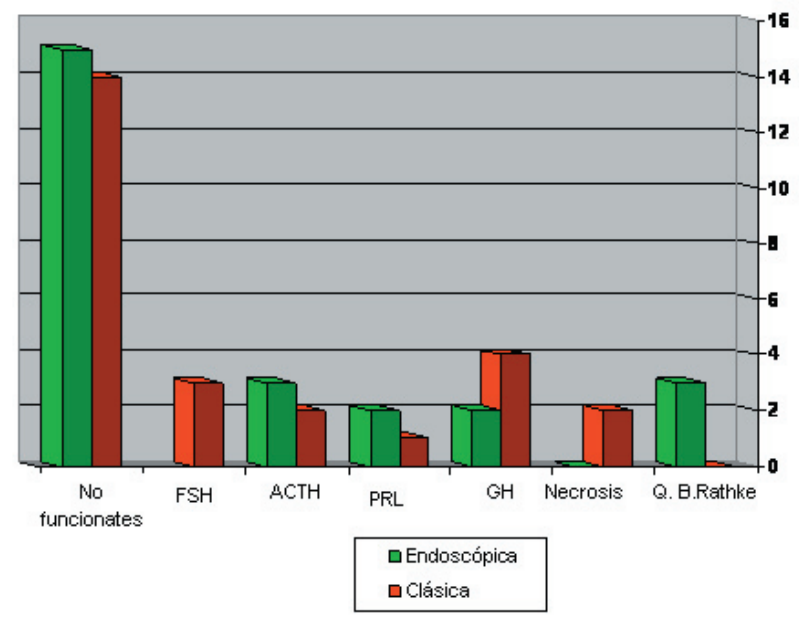

Gráfico 1. Características anatomo-patológicas.

\section{Resultados}

Los resultados clínicos en el grupo de la técnica clásica fueron: en 19 pacientes se consiguió una mejoría clínica $(82,6 \%)$. Se obtuvo una mejoría en todos los pacientes que presentaban déficit visual, se consiguió la curación/mejoría en dos acromegalias y en una enfermedad de Cushing. Fueron dos las acromegalias que continuaron igual (sin curación y con tratamiento médico), no mejoraron dos pacientes con impotencia, y la paciente que se diagnosticó por control clínico empeoró su clínica visual $(4,3 \%)$.

En el grupo endoscópico se obtuvo una mejoría en 21 pacientes (84\%) con curación de las dos enfermedades de Cushing y de las dos acromegalias. En 4 pacientes (16\%) no hubo cambios. Ningún paciente empeoró.

No existieron diferencias estadísticamente significativas entre el método quirúrgico empleado y la mejoría clínica $(\mathrm{p}=0,559)$.

En la tabla 1 se muestran las características clínicas de ambas series.

En el gráfico 1 podemos observar las características anatomo-patológicas de ambas series.

\section{Grado de resección}

En el grupo de abordaje clásico se consiguió una resección completa en 8 pacientes, una resección subtotal en 6, y una resección parcial en 9 (Gráfico 2). En el grupo endoscópico se consiguió en 15 pacientes una resección completa, en 8 una resección subtotal y en 2 una resección parcial. Ver Gráfico 3.

\section{Complicaciones}

En el grupo de técnica clásica un paciente fue éxitus. Dicho paciente presentó una fístula de LCR y le fue colocado un drenaje lumbar. El drenaje se retiró a los cinco días tras comprobar que había cesado la fístula. Los cultivos de LCR en todo momento fueron negativos y el paciente nunca estuvo febril. A los dos días, tras la retirada del drenaje 


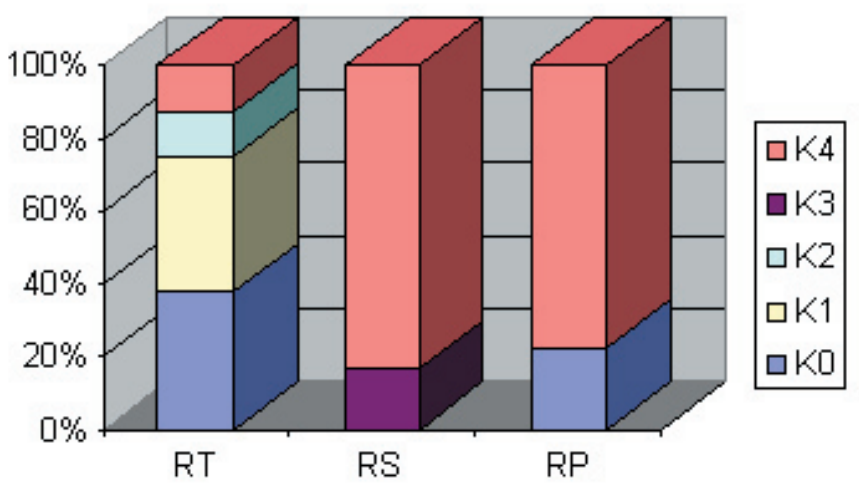

Gráfico 2. Grado de resección por técnica clásica en relación a los grados de invasividad.

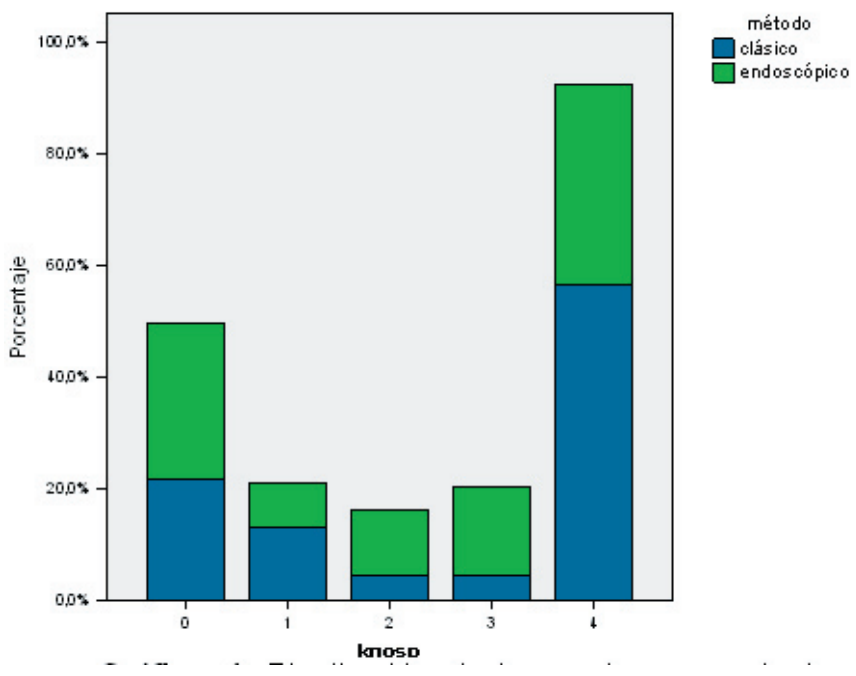

Gráfico 4. Distribución de los pacientes según la variable grados de Knosp y el método quirúrgico.

lumbar el paciente presentó una crisis epiléptica y midriasis bilateral arreactiva, se le colocó un drenaje ventricular y se extrajo LCR mostrando una meningitis pneumocócica fulminante sin respuesta a tratamiento médico.

Un paciente tuvo un empeoramiento de su visión con una hemianopsia bitemporal por lesión quiasmática.

Dos pacientes presentaron fístula de LCR, uno de ellos fue el outlier. Dicho paciente presentó una fístula de LCR en la que se colocó un drenaje lumbar que posteriormente se infectó, presentando una meningitis por E. coli que se complicó con una hidrocefalia. El otro paciente hizo una fístula LCR que se solucionó a los pocos días con el drenaje lumbar.

Un paciente con un macroadenoma grado IV realizó a las 12 horas de la cirugía un déficit completo del III pc que recuperó totalmente a los tres meses.

Siete pacientes $(30,4 \%)$ presentaron un panhipopituitarismo secundario a la cirugía, y no hubo ningún paciente

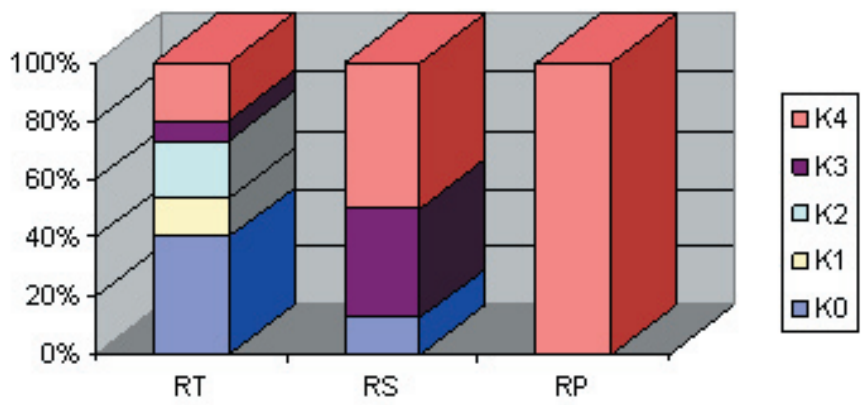

Gráfico 3. Grado de resección por técnica endoscópica en relación a los grados de invasividad.

con diabetes insípida permanente.

En el grupo endoscópico cuatro pacientes presentaron una fístula de LCR, estos pacientes fueron los primeros de la serie endoscópica que se intervinieron. A raíz de ello, se cambió la forma de cierre, pasando del taponamiento con grasa a no taponarlos. Con esta experiencia decidimos cambiar algunas pautas que comentaremos en la discusión.

Una paciente presentó una epistaxis a la semana que se solucionó con el ingreso y taponamiento.

Hormonalmente en un 16\% de los casos hubo panhipopituitarismo y en un $20 \%$ diabetes insípida.

\section{Relaciones entre el método quirúrgico, grado de resec- ción y estancia}

Asumiremos a lo largo de la investigación que ambos grupos presentaban unos grados de invasión tumoral similares $(p=0.408)$. Gráfico 4. La técnica endoscópica presenta un porcentaje de resección total muy superior al presentado por la técnica clásica (60\% frente a $34.8 \%$ ), un porcentaje mayor en cuanto a resección subtotal $(32 \%$ frente a $26.1 \%$ ) que la técnica clásica y un porcentaje muy inferior en cuanto a resección parcial (8\% frente a 39.1\%) que la técnica clásica. En contraposición, no se encontraron relaciones estadísticamente significativas entre el método de la intervención y lesión de los nervios ópticos, panhipopituitarismo, fístula de LCR, déficit de pares craneales, epistaxis, meningitis, diabetes, lesión de arteria carótida y control clínico.

En cuanto a la relación número de días de postoperatorio con el método quirúrgico aplicado, se ha identificado una relación estadísticamente significativa $(p=0.001)$ entre dichas variables. En este sentido, la media del número de días de postoperatorio para el grupo de pacientes intervenidos mediante la técnica clásica es de 6.61 (IC 95\% [5.67, 7.55]), mientras que para el grupo de pacientes operados mediante la técnica endoscópica la media es igual a 3.80 (IC 95\% [2.47, 5.13]). Así, podemos afirmar que el número medio de días de postoperatorio que pasa en el hospital un paciente que ha sido intervenido mediante la técnica clá- 
Tabla 2

Odds Ratio (OR) estimados para el éxito de la intervención (RX), respecto al método quirúrgico y grados de Knosp.

\begin{tabular}{|c|c|c|c|c|c|}
\hline & & $\begin{array}{c}\text { Éxito (Resección Total } \\
\text { / Subtotal) }\end{array}$ & $\begin{array}{l}\text { Poco Éxito } \\
\text { (RX parcial) }\end{array}$ & OR & IC 95\% \\
\hline \multirow[t]{4}{*}{ Método quirúrgico } & & $\mathrm{N}$ & $\mathrm{N}$ & & \\
\hline & Clásico & 14 & 9 & 1 & \\
\hline & Endoscópico & 23 & 2 & 0.157 & {$[0.028,0.866]$} \\
\hline & Total & 37 & 11 & & \\
\hline \multicolumn{6}{|l|}{ Grados Knosp 2} \\
\hline & $\begin{array}{l}\text { Leve/ } \\
\text { Moderado }\end{array}$ & 23 & 3 & 1 & \\
\hline & Grave & 14 & 8 & 3.595 & {$[0.750,7.230]$} \\
\hline & Total & 37 & 11 & & \\
\hline
\end{tabular}

sica es aproximadamente tres días superior a la media de días que pasa un paciente intervenido mediante la técnica endoscópica.

\section{Efecto del método quirúrgico con respecto al éxito de la operación}

En este sentido, el "éxito" de la intervención se encontrará asociado a los niveles resección total y subtotal, mientras que el "poco éxito" se encontrará ligado al nivel resección parcial. Adicionalmente, se introdujo en el modelo la variable grados Knosp 2, que mide el nivel de invasión de la enfermedad, y que permite controlar de alguna manera el factor "dificultad de la intervención quirúrgica". La Tabla 2 muestra las estimaciones para el riesgo de poco éxito de acuerdo al método quirúrgico aplicado y los grados de Knosp del paciente, categorizada esta última variable en dos únicos niveles: Leve/Moderado (de 0-3 en la escala original) y Grave (asociado al valor 4 de la escala original). En este sentido, cabe destacar que la interacción entre el método quirúrgico y el grado de invasión del tumor no es estadísticamente significativa. Lo mismo sucede con el efecto individual de la variable Knosp $2(p=0.110)$, que probablemente ha quedado encubierto como consecuencia del uso en la investigación de un tamaño muestral relativamente reducido. En cuanto al método quirúrgico empleado, éste sí es estadísticamente significativo $(p=0.034)$.

En relación a la estimación de los riesgos, el hecho de ser operado con el método endoscópico reduce en un $84 \%$ el riesgo de poco éxito en la intervención $(\mathrm{OR}=0.157$; $\mathrm{IC}$ $95 \%[0.028,0.866])$ con respecto a la aplicación de la técnica clásica. Y, por otra parte, se ha observado que un grado de invasión mayor del tumor hace incrementar 3.59 veces $(\mathrm{OR}=3.595$; IC 95\% $[0.750,17.230])$ el riesgo de poco éxito de la operación.

\section{Discusión}

El nivel de evidencia es bajo cuando intentamos comparar ambas técnicas, dado que no existen estudios prospectivos, a doble ciego y randomizados. La causa principal está en la dificultad de obtener por un solo centro un tamaño muestral adecuado, dada la incidencia de esta patología y la gran diversidad de tumores (secretores, no secretores y grados de invasividad) que implicarían una gran cantidad de categorías. Otro problema al comparar ambas técnicas surge dado que la técnica endoscópica es muy nueva, y el número de pacientes operados por la técnica clásica y su tiempo de seguimiento son mayores. Este trabajo va dirigido a evaluar cómo una técnica nueva puede mejorar los resultados de nuestros pacientes en un grupo que se está iniciando en cirugía hipofisaria.

Si analizamos la técnica microquirúrgica, los grados de resección tumoral dependen del tipo de tumor y del grado de invasividad. En los macroadenomas no funcionantes la resección completa va de un 29 a un $84 \%{ }^{8,13,33}$. Los índices de remisión más bajos se asocian a los adenomas gigantes invasivos ${ }^{16,17,27,28,37}$. Zada et al. ${ }^{38}$ a través de un abordaje endonasal microquirúrgico presentan unos resultados del 95, 40 y 33\% para macroadenomas no invasivos, macroadenomas invasivos y adenomas gigantes. En los prolactinomas los niveles de prolactina preoperatorios y la invasión dural son factores importantes. Zada et $\mathrm{al}^{38}$, refieren resecciones completas en un $75 \%$ en tumores con una PRL inferior a $200 \mathrm{ng} / \mathrm{ml}$ comparables al resto de los autores $^{35}$. En pacientes con enfermedad de Cushing con microadenomas los niveles de remisión se sitúan cerca 
del $100 \%$, siendo más bajos cuando no hay una imagen evidente preoperatoria, en macroadenomas invasivos $\mathrm{y}$ en las reintervenciones ${ }^{4,34,36}$. En pacientes con acromegalia la remisión es de un $75 \%$ en los microadenomas y de alrededor de un $50 \%$ en los macroadenomas ${ }^{38}$. Otras series presentan resultados similares ${ }^{1,15,25,32}$.

En el primer trabajo sobre endoscopia descrito por Jho y $\mathrm{Carrau}^{20}$ se describe una serie de 50 pacientes intervenidos a través de una fosa nasal. De los 8 pacientes con enfermedad de Cushing, 7 se curaron clínicamente y bioquímicamente. De los 17 prolactinomas, 10 mejoraron clínicamente y bioquímicamente, 4 mejoraron clínicamente pero continuaron con hiperprolactinemia y tres pacientes quedaron con restos en el seno cavernoso. Finalmente, de los 19 pacientes con macroadenomas no funcionantes en 16 se obtuvo una resección completa. El National Institute for clinical excellence interventional procedures programme, realizó un meta-análisis para evaluar la eficacia y seguridad de la técnica endoscópica respecto la clásica ${ }^{29}$. Realizaron una revisión en Febrero del 2003 en: Medline, Premedline, Embase, Cochrane Library and Science Citation Index. Las conclusiones del estudio fueron que en cuanto a la eficacia, los resultados (resección quirúrgica) eran comparables a la técnica convencional, si bien el tiempo quirúrgico y el postoperatorio eran inferiores (2-5 días a 4-10 días de la técnica clásica). Respecto a la seguridad, el tanto por ciento de complicaciones de la técnica endoscópica era inferior al de la clásica. Sólo en dos pacientes de 310 había aparecido una complicación grave como es la meningitis. Complicaciones leves fueron evidentes en un $7 \%$.

Otro grupo fundamental en la evolución de la endoscopia es el de E. de Divitiis y P. Cappabianca ${ }^{6}$. Describen una serie de 146 pacientes intervenidos desde enero del 1997 a julio de 2001. En los macroadenomas consiguieron una resección completa en 45 de los 80 no funcionantes, en 21 de los 34 tumores productores de GH y en cuatro de los siete macroprolactinomas. En los microadenomas se consiguió una resección completa en cinco de los seis secretores de $\mathrm{GH}$, en todos los microprolactinomas (6 pacientes) y en siete de los nueve secretores de ACTH. Un hecho a tener en cuenta al valorar los resultados en esta serie, es que el número de macroadenomas invasivos era bastante alto, que fueron los primeros 146 pacientes operados, y que 20 pacientes eran reintervenciones.

Frank y Pasquini ${ }^{6,14}$ en un estudio reciente de 2006 evalúan 418 pacientes con adenomas hipofisarios intervenidos entre 1998 y 2004. En esta serie, se consiguió la curación en los tumores secretores de GH (83\% microadenomas, $64,5 \%$ de los macroadenomas), en los prolactinomas $(85,7 \%$ de los microadenomas y $68,5 \%$ de los macroadenomas), en los secretores de ACTH $(67,7 \%$ de los microadenomas y $68 \%$ de los macroadenomas), y finalmente en los no funcionantes (61,1\% de los macroadenomas). El tiempo quirúrgico medio fue de 40 minutos, siendo éste parecido al de la técnica microquirúrgica. Sus conclusiones son que la técnica endoscópica, en cuanto a resultados es similar si nos referimos a tumores endoselares (respecto la técnica microquirúrgica), en cambio, es superior en tumores con extensión supraselar. Otros autores como Dehdashti ${ }^{11}$ han confirmado estos excelentes resultados posteriormente en series de 200 pacientes con un seguimiento medio de 19 meses.

En nuestra serie, se ha conseguido una resección total en un $60 \%$ de los casos con la técnica endoscópica, frente a un $34,8 \%$ con la técnica clásica, e igualmente la resección subtotal en la técnica endoscópica es de un 32\% frente al $26 \%$ de la técnica clásica. Cuando agrupamos y comparamos las variables (resección total más subtotal) frente a resección parcial $(<80 \%)$ vemos que existe una diferencia estadísticamente significativa $(p=0,033)$. Estos resultados se pueden atribuir al hecho de que el endoscopio nos ha permitido hacer una mejor exploración intraoperatoria del interior de la cavidad selar tras la exéresis tumoral. Con la técnica microscópica, es más difícil ver y valorar estos restos tumorales, y existen espacios no visibles donde hay que trabajar a ciegas como son los senos cavernosos y el espacio supraselar, siendo clave la experiencia del neurocirujano. Otro factor que apoya esta hipótesis es que el número de tumores altamente invasivos grados 3 y 4 de Knosp en las dos series es muy elevado en los dos grupos (14 pacientes $/ 60,8 \%$ clásica) y (13 pacientes $/ 52 \%$ endoscópica). Finalmente otro dato interesante y que confirma lo obtenido por otros autores ${ }^{29}$ es la disminución de la estancia postoperatoria. En nuestro caso existe una diferencia estadísticamente significativa $(\mathrm{p}=0,001)$. De hecho, un paciente intervenido por el método clásico permanecerá tres días más de estancia media en el hospital que uno operado vía endoscópica. Evidentemente estos datos son muy importantes por si mismos, ya que cualquier procedimiento que favorezca la precocidad en el alta lleva implícito una menor agresividad, y una mayor tolerancia y/o confortabilidad para el paciente. Por otra parte, el reducir la estancia hospitalaria permite reducir el gasto y favorecer la rotación cama/paciente, factor muy importante en una sanidad sobresaturada.

El abordaje transesfenoidal es un procedimiento razonablemente seguro y con una mortalidad baja, en la mayoría de las series inferior al 1\%3,8,26; sin embargo, la morbilidad no es despreciable ${ }^{8,26}$. De todas las series, como estándar utilizamos el trabajo de Ciric et al. ${ }^{8}$ donde este autor recoge la experiencia de 958 neurocirujanos y analiza la frecuencia de complicaciones en función del número de intervenciones (técnica microquirúrgica) practicadas por cirujano en total. Entre las causas más frecuentes de morbilidad destacan la fístula de LCR (1,5- 4.2\%), la diabetes insípida (7-20\%), los déficits de pares craneales $(0,4-$ 
$1,9 \%)$, la lesión de la arteria carótida $(0,4-1,4 \%)$, la pérdida de visión $(0,5-2,4 \%)$ meningitis $(0,5-1,9 \%)$, insuficiencia hipofisaria anterior $(7,2-20,6 \%)$, la perforación septal $(3,3-$ $7,6 \%)$, la epistaxis $(0,4-4,3 \%)$ y la sinusitis $(3,6-9,6 \%)^{5}$.

En el trabajo de Zada et al. ${ }^{38}$ de los 109 pacientes intervenidos, contaron con 7 complicaciones mayores y no hubo ningún éxitus. Setenta y ocho pacientes completaron un cuestionario donde se les preguntó por lo que peor habían percibido de todo el proceso quirúrgico, siendo en un 39\% el taponamiento nasal, en un 36\% la retirada del taponamiento y en un $35 \%$ el hecho de respirar por la boca. A partir de los tres meses cuantificaron que un $4 \%$ tenían dolor facial grave, congestión nasal grave un $3 \%$, importantes alteraciones del gusto y el olfato un $4 \%$ y finalmente un $1 \%$ presentaba anestesia importante del labio superior.

En otro trabajo realizado por Dusick $\mathrm{JR}^{12}$ con 259 pacientes intervenidos mediante técnica endonasal microquirúrgica, cabe destacar que sólo se realizó taponamiento en los primeros 95 pacientes. Un 73\% reconocían haber tenido una mejor experiencia de la que pensaban anteriormente, y un $8 \%$ peor. De los pacientes que habían respondido que la experiencia era peor, en un $15 \%$ se les habían colocado taponamiento. De los pacientes con cefalea preoperatoria; ésta se había resuelto en el $49 \%$ de los casos. Finalmente, los pacientes que habían sido sometidos anteriormente a un abordaje sublabial, preferían el abordaje endonasal porque se recuperaban mejor en un $87 \%$, tenían menos dolor en un $80 \%$ y menos obstrucción nasal en un $79 \%$.

$\mathrm{Si}$ revisamos las complicaciones en las series endoscópicas veremos que éstas suelen tener menor incidencia, principalmente a nivel seno facial. Otra característica es la ausencia de taponamiento, siendo un factor importante en la confortabilidad del paciente, como se desprende de los trabajos anteriormente mencionados. En 1999 Koren y et al. ${ }^{24}$ realizaban una comparación de las complicaciones endonasales entre el abordaje sublabial y el abordaje endoscópico. La técnica endoscópica estaba asociada a un menor tiempo quirúrgico, menor periodo postoperatorio, ausencia de epistaxis recurrente y de problemas dentales, menor incidencia de perforación septal, de sinequias y de formación de costras. En el trabajo de Cappabianca ${ }^{6}$ se clasifican y desglosan las complicaciones. Respecto a las complicaciones naso faciales únicamente destaca un caso de sinusitis (2\%); muy por debajo de las series clásicas, un $2 \%$ de fístulas de LCR, $0 \%$ de pérdida de visión, $0,68 \%$ de lesión arteria carótida, $0,68 \%$ de meningitis, $13,6 \%$ de déficit anterior hipofisario, $3,42 \%$ de diabetes insípida y un $0,68 \%$ de mortalidad.

En el meta-análisis realizado por el National Institute for clinical excellence interventional procedures progra$\mathrm{mme}^{29}$ concluyen que el porcentaje de complicaciones de la técnica endoscópica es más bajo que el de la clásica, siendo un procedimiento más seguro. Cho and $\mathrm{Liau}^{7}$ encuentran una diferencia estadísticamente significativa: $4,5 \%$ (endoscópica) y $27 \%$ (clásica). Budie ${ }^{2}$ refiere un $15 \%$ y un $24 \%$ respectivamente entre el grupo endoscópico y el clásico. En otros trabajos no hay diferencias ${ }^{21,31}$.

En la serie de Frank publicada en 2006 tienen un 0,7\% de complicaciones nasales (epistaxis), un 16\% de fístulas de LCR intraoperatorias y un $1,2 \%$ de postoperatorias, un $0,4 \%$ de meningitis, un $0,4 \%$ de empeoramiento visual, un $3,1 \%$ de insuficiencia hipofisaria anterior y un $7,6 \%$ de diabetes insípida.

En nuestra serie en el grupo de cirugía clásica, tres pacientes presentaron una fístula de LCR de los cuales dos de ellos se complicaron con meningitis. Uno de ellos falleció y un segundo presentó una hidrocefalia postmeningitis que después requirió una derivación ventrículoperitoneal. El fallecimiento del paciente de nuestra serie ocurrió una semana después de la intervención tras presentar una fístula de LCR que se complicó con una meningitis neumocócica fulminante. Sólo un paciente presentó un déficit del III par craneal postoperatorio que recuperó completamente a los pocos meses. En el grupo endoscópico la principal complicación fue la fístula $(16 \%)$ que sucedió en los primeros cuatro pacientes que intervinimos debido a la falta de experiencia con la técnica de cierre endoscópica. Si bien observamos que en nuestra serie no hay diferencias estadísticamente significativas entre ambos grupos, tenemos que hacer la crítica de que el bajo tamaño muestral puede haber influido de forma importante en este resultado. Sí nos parece interesante constatar que la aparición de la endoscopia ha cambiado la forma de manejar la fístula de LCR postoperatoria. En la serie clásica se opta por colocar un drenaje lumbar y dejar al paciente en reposo durante una semana. De hecho las dos complicaciones más importantes (éxitus e hidrocefalia) nacen de problemas con el drenaje lumbar. Actualmente en la serie endoscópica no hemos tenido ningún problema importante. Esto se debe a que si el paciente presenta una fístula LCR postoperatoria se opta por reintervenir al paciente las primeras $24-48 \mathrm{~h}$, así vemos con gran facilidad donde está el defecto y se tapona adecuadamente. Esta situación ha ido derivando hacia la no utilización de grasa, $y$, al no taponamiento de todo el seno. Actualmente realizamos una inspección de la silla, buscamos defectos, y si no hay, sólo utilizamos plastia dural. Sólo utilizaremos grasa y tissucol ${ }^{\circledR}$ cuando durante la cirugía se produzca una rotura del diafragma con salida importante de LCR. Destacar también, que con la técnica endoscópica tenemos mayor incidencia de diabetes insípida, derivado, en nuestra opinión, de una mayor agresividad en la resección tumoral. El resto de complicaciones son las esperables según la literatura. 


\section{Conclusiones}

En nuestra experiencia inicial la cirugía endoscópica endonasal nos ha permitido mejorar nuestros resultados y disminuir la estancia postoperatoria. No hemos observado diferencias en cuanto a complicaciones. Estudios con un mayor tamaño muestral son necesarios para obtener una mayor evidencia.

\section{Bibliografía}

1. Abosch, A., Tyrrell, J.B., Lamborn, K.R.: Transsphenoidal microsurgery for growth hormone-secreting pituitary adenomas: initial outcome and long term results. J Clin Endocrinol Metab 1998; 83: 3411-3418.

2. Badie, B., Nguyen, P., Preston, J.K.: Endoscopicguided direct endonasal approach for pituitary surgery. Surg Neurol 2000; 53: 168-172.

3. Black, P.M., Zervas, N.T., Candia, G.L.: Incidence and management of complications of transsphenoidal operation for pituitary adenomas. Neurosurgery 1987; 20: 920924.

4. Blevins, L.S., Jr., Christy, J.H., Khajavi, M., et al.: Outcomes of therapy for Cushing's disease due to adrenocorticotropin-secreting pituitary macroadenomas. J Clin Endocrinol Metab 1998; 83: 63-67.

5. Cappabianca, P., Alfieri, A., De Divitiis, E.: Endoscopic endonasal transsphenoidal approach to the sella: Towards functional endoscopic pituitary surgery. Minim Invasive Neurosurg 1998; 41: 66-738.

6. Cappabianca, P., Cavallo, L.M., Colao, A.M. et al.: Surgical complications associated with the endoscopic endonasal transsphenoidal approach for pituitary adenomas. J Neurosurg 1997; 97: 293-298.

7. Cho, D.Y. Liau, W.R.: Comparison of endonasal endoscopic surgery and sublabial microsurgery for prolactinomas. Surg Neurol 2002; 58: 371-376.

8. Ciric, I., Ragin, A., Baumgartner, C., Pierce, D.: Complications of transsphenoidal surgery: results of a national survey, review of the literature, and personal experience. Neurosurgery 1997; 40: 225-236.

9. Cooke, R.S. Jones, R.A.: Experience with the direct transnasal approach to the pituitary fossa. $\mathrm{Br} \mathrm{J}$ Neurosurg 1994; 8: 193-196.

10. de Diviitis, E., Cappabianca, P., Cavallo, L.M.: Endoscopic endonasal transsphenoidal approach surgery. Adv Tech Stand Neurosurg 2002; 27: 137-177.

11. Dehdashti, A.R., Ganna, A., Karabatsou, K., Gentili, F.: Pure endoscopic endonasal approach for pituitary adenomas: early surgical results in 200 patients and comparison with previous microsurgical series. Neurosurgery 2008; 62: 10061015.

12. Dusick, J.R., Esposito, F., Mattozo, C.A., et al.:
Endonasal transsphenoidal surgery: the patient's perspectivesurvey results from 259 patients. Surg Neurol 2006; 58: 857865.

13. Ebersold, M.J., Quast, L.M., Laws, E.R., Jr., et al.: Long-term results in transsphenoidal removal of nonfunctionating pituitary adenomas. Journal of Neurosurgery 1986; 64: 713-719.

14. Frank, G., Pasquini, E., Farneti, G., et al.: The endoscopic versus the traditional approach in pituitary surgery. Neuroendocrinology 2006; 83: 240-248.

15. Freda, P.U., Wardlaw, S.L., Post, K.D.: Long-term endocrinological follow-up evaluation in 115 patients who underwent transsphenoidal surgery for acromegaly. J Neurosurg 1998; 89: 353-358.

16. Garibi, J., Pomposo, I. Villar, G., et al.: Giant pituitary adenomas: clinical characterisitcs and surgical results. $\mathrm{Br} \mathrm{J}$ Neurosurg 2002; 16: 133-139.

17. Goel, A. Nadkarni, T.: Surgical management of giant pituitary tumors-a review of 30 cases. Acta Neurochir 1996; 138: 1042-1049.

18. Guiot, G., Rougerie, J., Fourestier, M., et al.: Intracranial endoscopic explorations. Presse Med 1963; 71: 12251228.

19. Hazan, A., Roux, F.X., Levy, D., et al.: Use of endoscopy in pituitary surgery. Neurochirurgie 1998; 44: 327330 .

20. Jho, H.D. Carrau, R.L.: "Endoscopic endonasal transsphenoidal surgery: experience with 50 patients.". J Neurosurg 1997; 87: 44-51.

21. Kawamata, T., Iseki, H., Ishizaki, R., Hori, T.: Minimally invasive endoscope-assisted endonasal transsphenoidal microsurgery for pituitary tumors: experience with 215 cases comparing with sublabial transsphenoidal approach. Neurol Res 2002; 24: 259-265.

22. Kawamata, T., Kamikawa, S., Iseki, H., et al.: Flexible endoscope-assisted endonasal transsphenoidal surgery for pituitary tumors. Minim Invasive Neurosurg 2002; 45: 208210 .

23. Knosp, E., Steiner, E., Kitz, K., Matula, C.: Pituitary adenomas with invasion of the cavernous sinus space: a magnetic resonance imaging classification compared with surgical findings. Neurosurgery 1993; 33: 610-617.

24. Koren, I., Hadar, T., Rappaport, Z.H., Yaniv, E.: Endoscopic transnasal transsphenoidal microsurgery versus the sublabial approach for the treatment of pituitary tumors: endonasal complications. Laryngoscope 1999; 109: 18381840 .

25. Kreutzer, J., Vance, M.L., Lopes, M.B., et al: Surgical management of GH-secreting pituitary adenomas: an outcome study modern remission criteria. J Clin Endocrinol Metab 2001; 86: 4072-4077.

26. Laws, E.R., Jr. Kern, E.B.: Complications of trans- 
sphenoidal surgery. Clin Neurosurg 1976; 23: 401-416.

27. Meij, B.P., Lopes, M.B., Ellegala, D.B., et al.: The long term significance of microscopic dural invasion in 354 patients with pituitary adenomas treated with transsphenoidal surgery. J Neurosurg 2002; 195-208.

28. Mohr, G., Hardy, J., Comtois, R., Beauregard, H.: Surgical management of giant pituitary adenomas. Can J Neurol Sci 1990; 17: 62-66.

29. National Institute for Clinical Excellence. Endoscopic transsphenoidal pituitary adenoma ressection. 2007. Ref Type: Unpublished Work

30. Rodziewicz, G.S. Kelley, R.T. K.R.: Transnasal endoscopic surgery of the pituitary gland: Technical note. Neurosurgery 1996; 39: 649-652.

31. Sheehan, M.T., Atkinson, J.L.D., Kasperbauer, J.L., et al.: Preliminary comparison of the endoscopic transnasal vs the sublabial transseptal approach for clinically nonfunctioning pituitary macroadenomas. Mayo Clinic Proceedings 1999; 74: 661-670.

32. Shimon, I., Cohen, Z.R., Ram, Z.: Transsphenoidal surgery for acromegaly: endocrinological follow-up of 98 patiens. Neurosurgery 2001; 48: 1239-1245.

33. Tang, G., Ning, N.M., Nyugen, M.H., et al.: Endocrine-inactive pituitary adenomas: clinicopathological features and log term outcome following transsphenoidal resection. J Neurosurg 1998; 88: 393A (Abstract).

34. Tindall, G.T., Herring, C.J., Clark, R.V., et al.:

\section{Comentario al trabajo Comparación del abordaje sublabial transesfenoidal microquirúrgico frente al abordaje endonasal transesfenoidal endoscópico. Estu- dio prospectivo de 50 pacientes de J. Enseñat y cols.}

En el presente trabajo, los autores llevan a cabo una acertada comparación entre las dos técnicas que en el momento actual coexisten y compiten de cara a la resección de los tumores hipofisarios: la microcirugía y la endoscopia. La técnica microquirúrgica convencional es un procedimiento muy fiable y claramente consagrado que ha demostrado sus virtudes durante las pasadas décadas. La técnica endoscópica ha irrumpido con fuerza en esta parcela de la patología basicraneal, aunque se han abierto no pocos interrogantes respecto a sus ventajas reales en el día a día para la mayoría de pacientes. Para los autores, en el presente trabajo se pone de manifiesto que la técnica endoscópica consigue un mayor grado de resección con
Cushing's disease: results of transsphenoidal microsurgery with emphasis on surgical failures. J Neurosurg 1990; 72: 363-369.

35. Tyrrell, J.B., Lamborn, K.R., Hannegan, L.T., et al.: Transsphenoidal microsurgery therapy of prolactinomas : inicial outcomes and long-term results. Neurosurgery 1999; 44: 254-263.

36. Watson, J.C., Shawker, T.H., Nieman, L.K., et al.: Localization of pituitary adnomas by using intraoperative ultrasound in patients with Cushing's disease and no demostrable pituitary tumor on magnetic resonance imaging. J Neurosurg 1998; 89: 927-932.

37. Yokoyama, S., Hirano, H., Moroki, K., et al: Are nonfunctioning pituitary adenomas extending into the cavernous sinus aggressive and/or invasive? Neurosurgery 2001; 49: 857-862.

38. Zada, G., Kelly, D.F. Cohan, P., et al.: Endonasal transsphenoidal approach for pituitary adenomas and other sellar lesions. an assessment of efficacy, safety, and patient impressions. J Neurosurg 2003; 98: 350-358.

Enseñat, J.; Quesada, J.L.; Aparicio, J.; Pàmies, C.; Barber, X.; Topczewski, Th.; Ferrer, E.: Comparación del abordaje sublabial transesfenoidal microquirúrgico frente al abordaje endonasal transesfenoidal endoscópico. Estudio prospectivo de 50 pacientes. Neurocirugía 2009, 20: $335-345$ una morbilidad similar y un mayor confort para el paciente, argumentos más que convincentes para contribuir a estimular a la comunidad neuroquirúrgica (en particular a la que dirige mayor dedicación a patología hipofisaria y basicraneal) a profundizar en esta prometedora técnica. Sólo cabe objetar el cierto sesgo que supone que lógicamente la primera parte de la serie (microquirúrgica) se entiendo corresponde quizá a la primera parte de la curva de aprendizaje, no ya en una técnica en sí, si no en el manejo de la patología hipofisaria en sí. Salvado este aspecto, el trabajo constituye un estimulante motivo para reflexionar acerca de la utilidad potencial de la técnica no sólo para la patología tumoral hipofisaria, sino para todas aquellas patologías 
que se vienen abordando mediante abordajes transorales y transfaciales, de mayor agresividad y con campo de visión "microquirúrgico" mucho más limitado que el endoscópico.

\section{Bibliogafía}

Fatemi, N., Dusick, J.R., de Paiva Neto, M.A., Kelly, D.F.: The endonasal microscopic approach for pituitary adenomas and other parasellar tumors: a ten years experience. Neurosurgery 2008 Oct; 63 (4 Suppl 2): 244-256

M.A. Arráez

Málaga 